\title{
Experimental Drugs for the Treatment of Hepatitis D
}

This article was published in the following Dove Press journal:

Journal of Experimental Pharmacology

\author{
Lisa Sandmann' \\ Markus Cornberg $\mathbb{1 D}^{1,2}$ \\ 'Department of Gastroenterology, \\ Hepatology and Endocrinology, Hannover \\ Medical School, Hannover, Germany; \\ ${ }^{2}$ Centre for Individualised Infection \\ Medicine (CiiM), Helmholtz Centre for \\ Infection Research, Braunschweig, \\ Germany
}

\begin{abstract}
Chronic hepatitis D virus infection is the most severe form of viral hepatitis. Antiviral treatment is urgently needed to prevent patients from developing end stage liver disease or hepatocellular carcinoma. Treatment options were limited to off-label use of pegylated interferon alfa until conditional approval of bulevirtide by the EMA (European Medicines Agency) in July 2020. However, several other antiviral compounds are currently investigated and represent promising agents for the treatment of chronic HDV infection.
\end{abstract}

Keywords: hepatitis delta, HBV/HDV coinfection, interferon, bulevirtide, lonafarnib

\section{Introduction}

Chronic Hepatitis D Virus (HDV) infection, also known as hepatitis delta, is the most severe form of viral hepatitis. ${ }^{1}$ Approximately $5 \%$ of all HBsAg positive patients are co-infected with HDV leading to 10-12 million cases worldwide. ${ }^{2}$ However, hyperendemic regions experience higher rates of co-infection and screening rates may vary in different areas of the world. Chronic HDV infection (CHD) is associated with a 3.8-fold higher risk of developing liver cirrhosis compared to patients infected with hepatitis B virus (HBV) alone and patients experience a 1.28fold higher risk for the development of hepatocellular carcinoma (HCC). ${ }^{3,4}$ All in all, the 5-year mortality rate is increased in patients with HBV/HDV coinfection compared to HBV mono-infected patients. ${ }^{5,6}$

Due to its progressive course, antiviral therapy for the treatment of HDV infection is urgently needed. Pegylated interferon alfa (PEG-IFN- $\alpha$ ) is approved for the treatment of $\mathrm{HBV}$ and is also effective against HDV. In the two large controlled prospective HIDIT studies, the response rate was $23-48 \%$ at the end of therapy. ${ }^{7,8}$ However, twenty-four weeks after the end of therapy only about $25 \%$ of patients were HDV RNA negative and in the further long term about $50 \%$ of these patients had a late HDV-RNA relapse. ${ }^{9}$ Despite its limited long-term virological response rates, antiviral treatment with PEGIFN- $\alpha$ is associated with a lower risk of developing clinical complications and maintained virological response is even more strongly linked to a more favorable long-term course. ${ }^{10-12}$ Nevertheless, IFN-based antiviral treatment is accompanied by severe side effects, treatment is limited to patients with compensated liver disease and the aspired maintained virological response is rare. Therefore, there is an urgent need for new therapeutic approaches and several substances are currently evaluated in clinical phase II and III trials. Recently, the entry-inhibitor bulevirtide has received conditional approval by EMA while results of the ongoing phase III study are still pending. The prenylation inhibitor lonafarnib has entered phase III clinical trials. In addition, nucleic acid
Correspondence: Markus Cornberg Hannover Medical School, Department of Gastroenterology, Hepatology and Endocrinology, Carl-Neuberg-Str. I, Hannover, 30625, Germany Tel +495II 532682 I

Email cornberg.markus@mh-hannover.de
Journal of Experimental Pharmacology 2021:13 46|-468 
polymers, RNA interference compounds and interferon lambda are other potential substances currently being tested. Importantly, treatment with nucleot(s)ide analogues (NA), which inhibit reverse transcription of $\mathrm{HBV}$, have no direct effect on HDV replication. Nevertheless, NA are considered in terms of liver disease severity and HBV DNA levels in the therapy of patients with HBV/HDV coinfection. ${ }^{13}$ An overview of therapeutic approaches is depicted in Figure 1 and detailed information on the different substances is provided in the following.

\section{Parameters for Treatment Response in Hepatitis Delta}

The aim of antiviral treatment of viral hepatitis is the prevention of hepatic complications like liver cirrhosis, hepatic decompensation, development of HCC and liverrelated death. Surrogate markers to predict improved clinical outcome and therefore defining treatment response are still a matter of debate for CHD. In HBV mono-infection long-term suppression of HBV DNA is associated with a reduced risk of developing liver-related complications. ${ }^{13}$ Similarly, suppression of HCV RNA replication in patients chronically infected with $\mathrm{HCV}$ leads to better overall survival. ${ }^{14}$ For CHD, declines in HDV RNA during IFN$\alpha$ treatment have been associated with improved clinical outcome even if HDV RNA negativity could not be achieved. ${ }^{10,11}$ Therefore, a group of experts recently suggested an HDV RNA decline of $\geq 2 \log$ at the end of treatment as a surrogate parameter for treatment efficacy in CHD. ${ }^{15}$ However, HDV RNA typically fluctuates over

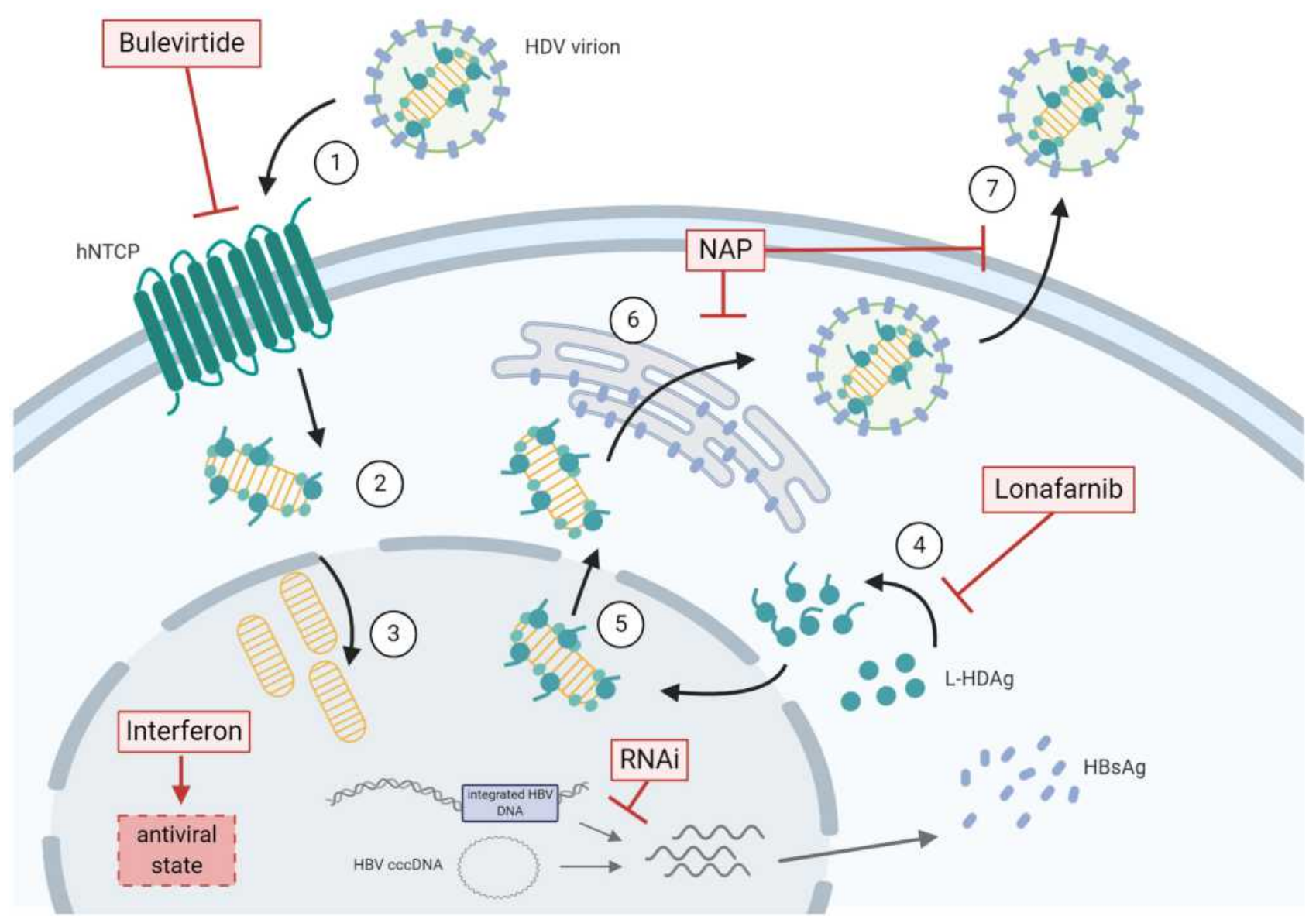

Figure I Therapeutic approaches for the treatment of chronic HDV infection are depicted in relation to the viral life cycle. (I) HDV virions attachment to heparan sulfate proteoglycans and binding of L-HBsAg pre-SI region to the HBV/HDV specific receptor, hNTCP. Viral particles enter the cell through endocytosis and the viral ribonucleoprotein (RNP) is released in the cytoplasm. (2) Viral RNP translocation to the nucleus. (3) HDAg mRNA transcription and replication of HDV RNA. (4) L-HDAg contains a prenylation site that is farnesylated by a cellular farnesyltransferase before being re-translocated to the nucleus. (5) Both forms of HDAg interact with the newly synthesized genomic RNA to form new viral (RNP) that are exported to the cytoplasm. (6) Viral RNPs interact with the cytosolic part of HBsAg at the endoplasmic reticulum surface inducing their envelopment. (7) HDV virions are secreted form the infected cell. The different steps targeted by antiviral treatments are depicted. HBV coinfection is represented by presence of HBV cccDNA and integrated HBV DNA. The figure was created with BioRender.com.

Abbreviations: cccDNA, covalently closed circular DNA; HBsAg, hepatitis B surface antigen; HDV, hepatitis D virus; hNTCP, human sodium taurochlorate co-transporting polypeptide; L-HDAg, large hepatitis D antigen; NAP, nucleic acid polymers; RNAi, RNA interference compounds. 
time which creates uncertainty regarding the finality of this endpoint. Several secondary end-points like early virological response, biochemical response (ALT normalization at the end of treatment/during follow up), histological evaluation and changes of HBsAg are additional parameters, which should be assessed during treatment. Ideally, the endpoint of finite antiviral treatment would be the loss of HBsAg, which corresponds to functional cure in chronic HBV infection and would also result in the inability for HDV assembly, release and transmission. To achieve HBsAg clearance, it is important to consider that both cccDNA and integrated HBV DNA may be the source of HBsAg (Figure 1) and therefore must be addressed by either antiviral or immunomodulatory therapies. Since the endpoint HBsAg clearance is still very rarely achieved, negative HDV RNA 24 weeks after stopping antiviral treatment has been suggested as the endpoint of finite treatment strategies. ${ }^{16}$

\section{Therapeutic Agents}

In Figure 1 different therapeutic approaches for the treatment of CHD are depicted. Beside interferon alfa, the by far longest known agent, new substances and their mode of actions are illustrated. Table 1 provides an overview of antiviral compounds currently investigated in clinical trials.

\section{Interferon Alfa}

Since the 1980, interferon alfa, and later pegylated IFN- $\alpha$, has been used as an off-label treatment of CHD. In the two largest prospective clinical trials viral response rates range between 25 to $30 \%$ without any clear additional benefit of concomitant treatment with nucleotide analogues. ${ }^{7,8}$ Virological response rates are further reduced since late relapses occur in up to $50 \%$ of patients with negative HDV RNA at the end of treatment. ${ }^{9}$ However, an antiviral treatment course might be worthy since HDV RNA suppression or decline due to treatment with PEG-IFN- $\alpha$ is associated with improved clinical long-term outcome according to several published studies. ${ }^{10,11,17}$

Positive long-term effects have to be weighed up against side effects like flu-like symptoms, anemia and thrombocytopenia or psychiatric disturbances which frequently occur during treatment. In addition to that, treatment is contraindicated in patients with advanced chronic liver disease therefore excluding many CHD patients urgently in need of antiviral treatment.
Treatment duration in most of the studies was one year and extension to 96 weeks did not improve virological response rates in the majority of patients. ${ }^{8}$ Therefore, international guidelines recommend a treatment duration of 48 weeks for PEG-IFN- $\alpha{ }^{13}$ An extension of treatment duration can be discussed if patients show an HBsAg decline assuming HBsAg loss during prolonged treatment. However, HBsAg loss only occurred in $<10 \%$ of the patients during the large clinical trials and therefore remains a rare event. ${ }^{7,8}$ HDV RNA negativity at treatment week 24 was associated with negative HDV RNA 24 weeks after the end of treatment and could be utilized as a parameter for treatment management. ${ }^{18}$

\section{Bulevirtide}

Binding of HBsAg to the sodium taurochlorate cotransporting polypeptide (NTCP) is crucial for $\mathrm{HBV} /$ HDV entry into hepatocytes. ${ }^{19}$ Bulevirtide (former Myrcludex B) is a small peptide blocking the engagement of the HBsAg preS1 domain with NTCP. Therefore, de novo infection of former uninfected hepatocytes is prohibited and over time recolonization of the liver with uninfected cells could finally lead to elimination of HDV.

In the first phase IIa study, 24 patients were included and equally randomized to receive (I) bulevirtide $2 \mathrm{mg}$ (daily) alone for 24 weeks followed by PEG-IFN- $\alpha 180 \mu \mathrm{g}$ (weekly) for 48 weeks or (II) the combination of bulevirtide $2 \mathrm{mg}$ (daily) and PEG-IFN- $\alpha 180 \mu \mathrm{g}$ (weekly) for 24 weeks followed by PEG-IFN- $\alpha$ monotherapy (weekly) for 24 weeks or (III) PEG-IFN- $\alpha$ alone for 48 weeks. HDV RNA declined significantly in all treatment groups at week 24. Monotherapy with bulevirtide was associated with HDV RNA decline in all patients and two patients became HDV RNA negative at week 24. Interestingly, combination with PEG-IFN- $\alpha$ increased the number of HDV RNA negative patients at week 24 as five patients of treatment group II showed negative HDV RNA. Furthermore, quantitative HDV RNA decline was more pronounced in the combination group. None of the patients achieved the primary endpoint of $>0.5 \log$ reduction of $\mathrm{HBsAg}$ at treatment week $12 .^{20}$

In the following dose-finding phase IIb study, 120 patients were assigned to either receive bulevirtide $2 \mathrm{mg}$, $5 \mathrm{mg}$ or $10 \mathrm{mg}$ in combination with tenofovir daily or tenofovir daily alone for 24 weeks. $^{21}$ The primary endpoint of $>2 \log$ HDV RNA decline at the end of treatment was achieved in 46,47 and $77 \%$ of patients with escalating doses of bulevirtide as compared to $3 \%$ in the TDF 
Table I New Drugs for Antiviral Treatment in Chronic HDV Infection

\begin{tabular}{|c|c|c|c|}
\hline Compound & Target & Phase of Development & Comments/Data \\
\hline $\begin{array}{l}\text { Bulevirtide (entry } \\
\text { inhibitor) }\end{array}$ & $\begin{array}{l}\text { NCTP (Sodium taurocholate co-transporting } \\
\text { polypeptide) receptor antagonist }\end{array}$ & $\begin{array}{l}\text { Phase III } \\
\text { Conditional approval by EMA }\end{array}$ & $\begin{array}{l}\text { - Subcutaneous application } \\
\text { - Overall, well tolerated } \\
\text { - Increase of bile acids, local side reactions } \\
\text { - Monotherapy: Decline of serum HDV RNA and ALT } \\
\text { - normalization, no effect on } \mathrm{HBsAg}^{21} \\
\text { - Combination (pegIFNa): stronger effect on HDV RNA } \\
\text { and } \mathrm{HBsAg}^{22}\end{array}$ \\
\hline $\begin{array}{l}\text { Lonafarnib } \\
\text { (prenylation } \\
\text { inhibitor) }\end{array}$ & Farnesyltransferase inhibitor & Phase III & $\begin{array}{l}\text { - Oral application } \\
\text { - Boosting with ritonavir reduces side effects (GI side } \\
\text { effects) } \\
\text { - Monotherapy: HDV RNA decline, no effect on HBsAg } \\
\text { - Combination (pegIFNa): stronger effect on HDV RNA } \\
\text { - Viral and biochemical flares post-treatment are asso- } \\
\text { ciated with HDV RNA and ALT response }{ }^{28}\end{array}$ \\
\hline $\begin{array}{l}\text { Pegylated } \\
\text { interferon-lambda }\end{array}$ & $\begin{array}{l}\text { Immune modulation } \\
\text { Induction of ISG }\end{array}$ & Phase II & $\begin{array}{l}\text { - Subcutaneous application } \\
\text { - Less side effects than pegIFNa } \\
\text { - ALT flares on treatment }{ }^{35}\end{array}$ \\
\hline $\begin{array}{l}\text { REP } 2139 \text { (nucleic } \\
\text { acid polymers) }\end{array}$ & $\begin{array}{l}\text { Amphipathic alpha-helices in Class I surface } \\
\text { glycoproteins }\end{array}$ & Phase II & $\begin{array}{l}\text { - Intravenous application } \\
\text { - ALT flares in combination with TDF and pegIFNa } \\
\text { - Strong effect on HDV RNA and } \mathrm{HBsAg}^{38} \\
\text { - So far only data from } 12 \text { patients }\end{array}$ \\
\hline JNJ-3989 & Ribonucleic acid interference & Phase II & $\begin{array}{l}\text { - Subcutaneous application } \\
\text { - Injection side irritations } \\
\text { - No results regarding HDV treatment so far }\end{array}$ \\
\hline
\end{tabular}

monotherapy group (only one patient). More than $40 \%$ of patients treated with bulevirtide achieved ALT normalization but no dose depending effect could be detected as 43 , 50 and $40 \%$ of the patients receiving bulevirtide $2 \mathrm{mg}$, $5 \mathrm{mg}$ and $10 \mathrm{mg}$ reached this secondary endpoint, respectively. An effect on HBsAg levels could not be revealed in any of the treatment groups and HDV RNA relapse after end of treatment occurred in most of the patients. Overall, treatment with bulevirtide was well tolerated: local reactions at the injection site and mild and transient neutropenia, thrombocytopenia and eosinophilia were observed. Elevation of bile salts did not lead to clinical symptoms.

In an additional multicenter phase IIb study prolonged treatment duration with bulevirtide at different dosages alone or in combination with PEG-IFN- $\alpha$ was assessed. ${ }^{22}$ 60 patients were randomly assigned to receive (I) PEGIFN- $\alpha 180 \mu \mathrm{g}$, (II) bulevirtide 2 mg plus PEG-IFN- $\alpha 180$ $\mu \mathrm{g}$, (III) bulevirtide $5 \mathrm{mg}$ plus PEG-IFN- $\alpha 180 \mu \mathrm{g}$ or (IV) bulevirtide $2 \mathrm{mg}$ for 48 weeks. Median HDV RNA reduction at the end of treatment was higher in the combination therapy groups as compared to PEG-IFN- $\alpha$ or bulevirtide $2 \mathrm{mg}$ alone. Nevertheless, HDV RNA and ALT levels showed a linear decline in the bulevirtide monotherapy group during treatment. Similar to the previous phase II study, stopping of bulevirtide treatment caused HDV RNA relapse in $>2 / 3$ of the patients. Interestingly, $53.3 \%$ and $26.7 \%$ of the patients receiving bulevirtide $2 \mathrm{mg}$ plus PEG-IFN- $\alpha$ or bulevirtide $5 \mathrm{mg}$ plus PEG-IFN- $\alpha$ remained HDV RNA negative at 24 weeks after the end of treatment, respectively. HBsAg response defined as $\geq 1 \log 10$ decline or negativity at 24 weeks after the end of treatment was detected in $46.7 \%$ of the patients treated with bulevirtide $2 \mathrm{mg}$ plus PEG-IFN- $\alpha$ and in $20 \%$ receiving bulevirtide $5 \mathrm{mg}$ plus PEG-IFN- $\alpha$. None of the patients receiving bulevirtide or PEG-IFN- $\alpha$ monotherapy achieved this endpoint. In an extension study of this study patients received high dose bulevirtide $(10 \mathrm{mg})$ either in combination with PEG-IFN- $\alpha$ or tenofovir. The primary end point of HDV RNA negativity 24 weeks after the end of treatment was achieved in $6.7 \%$ of the patients receiving bulevirtide $10 \mathrm{mg}$ plus PEG-IFN- $\alpha$ and in $33.3 \%$ of the patients receiving the combination with tenofovir. ${ }^{23}$ None of the patients receiving bulevirtide plus tenofovir achieved HBsAg decline or negativity whereas one patient $(6.7 \%)$ 
of the bulevirtide $10 \mathrm{mg}$ plus PEG-IFN- $\alpha$ group remained HBsAg negative 24 weeks after the end of treatment.

Overall, bulevirtide showed a dose-dependent antiviral effect regarding HDV RNA decline in the monotherapy trials. In combination with PEG-IFN- $\alpha$ the dosedependent effect on HDV RNA and HBsAg decline reversed: The effect on HBsAg seemed to be more pronounced in the groups receiving lower doses of bulevirtide in combination with PEG-IFN- $\alpha$. The reasons for this observation are not clear yet. However, bulevirtide and interferon show a synergistic antiviral effect in the treatment of CHD.

The results of the phase II studies, the limited therapeutic options for the treatment of CHD and its status of orphan disease finally led to the conditional approval of bulevirtide by the EMA (European Medical Agency) in July 2020. While phase III studies are still ongoing, patients with CHD, positive HDV RNA and compensated liver disease can now be treated with bulevirtide $2 \mathrm{mg}$ as a monotherapy in the European Union. Duration of treatment is so far not defined. According to the EMA approval, treatment should be maintained as long as patients show a benefit. ${ }^{24}$ Some real-world experiences showed an ongoing biochemical and virological response in patients with long-term administration. However, data on prolonged treatment is still scarce. ${ }^{25}$

\section{Lonafarnib}

Farnesylation of the large HD antigen is mandatory for HDV virion assembly. ${ }^{26}$ Inhibitors of cellular farnesylation disrupt viral assembly and therefore reduce the release of functional HDV virions. The farnesyl transferase inhibitor lonafarnib has been studied in phase I and II studies, and phase III studies are currently ongoing. ${ }^{27,28}$ Lonafarnib was tested as monotherapy, in combination with ritonavir (to increase lonafarnib levels by inhibition of cytochrome P450 3A4, the predominant mediator of lonafarnib metabolism) and with PEG-IFN- $\alpha$. Results from the pilot study (LOWR HDV-1 study) showed that higher doses of lonafarnib were associated with a stronger decrease of HDV RNA. However, with increasing doses, adverse events, mainly gastrointestinal side effects like diarrhea and nausea, increased as well. The combination with ritonavir allowed lower lonafarnib doses, which resulted in better virological effects with lower rates of adverse events and a similar effect was observed in combination with PEG-IFN- $\alpha .^{28}$ The optimal treatment regimen was explored in the subsequent LOWR HDV-2 study. ${ }^{29}$ Fifty-five patients with compensated liver disease were included and randomized to either receive high ( $\geq 75 \mathrm{mg}$ ), medium (50 $\mathrm{mg}$ ) or low (25 mg) doses of lonafarnib twice daily (bid) in combination with ritonavir or as a triple therapy in combination with PEG-IFN- $\alpha$ for 6 months. High rates of gastrointestinal side effects were observed in the high dose treatment arm. The primary end point of $>2 \log$ reduction of HDV RNA or negative HDV RNA at the end of treatment was achieved in 6 of 12 patients $(50 \%)$ in the all-oral combination of lonafarnib $50 \mathrm{mg}$ bid plus ritonavir treatment group while patients receiving $25 \mathrm{mg}$ showed lower response rates. Triple therapy in combination with PEGIFN- $\alpha$ achieved the best response rates and 8 of 9 patients showed a $>2 \log$ reduction of HDV RNA at the end of treatment. In a dose escalation study, the maximum dose of lonafarnib $100 \mathrm{mg}$ bid plus ritonavir was tolerated by two third of the patients (10/15). At the end of the 24week treatment, one patient showed negative HDV RNA and one patient had HDV RNA levels below the lower limit of detection. Liver enzymes were normalized in half of the patients. ${ }^{30}$ An additional study evaluated oncedaily dosing of lonafarnib in combination with ritonavir. After 24 weeks of treatment with either 50,75 or $100 \mathrm{mg}$ lonafarnib boosted with ritonavir, six of 21 patients showed HDV RNA levels $<250 \mathrm{IU} / \mathrm{mL}^{31}$ Some patients showed post-treatment viral and biochemical flares, which were associated with future HDV RNA negativity, normalization of liver enzymes and in some cases suppression of HBV DNA. The mechanism of these responses after ending lonafarnib treatment is unclear but an immunological mechanism is suggested. The pronounced gastrointestinal side effects at high dosages, mainly nausea, diarrhea, anorexia and weight loss, are significantly reduced for dosages of 50 or $100 \mathrm{mg}$ per day.

All in all, the available studies show that treatment with lonafarnib leads to HDV RNA decline and normalization of liver enzymes in patients with HDV infection. Boosting with ritonavir allows dose reduction of lonafarnib to reduce gastrointestinal side effects while maintaining antiviral efficacy. To date, the combination with PEGIFN- $\alpha$ achieves the best response rates but results from larger clinical trials are still lacking. A phase III clinical trial has been initiated. In addition, lonafarnib is also evaluated in combination with pegylated interferon lambda (see below). 


\section{Pegylated Interferon Lambda}

Pegylated interferon lambda (PEG-IFN-lambda) belongs to the type III interferons, which stimulate cell-mediated immune responses during viral infections. PEG-IFNlambda binds to type III IFN receptors resulting in activation of the Jak-STAT signaling pathway, similar to IFNalfa complexes. Type III interferon receptors are mainly expressed in hepatocytes and to a lesser extend in hematopoietic and central nervous system cells. Therefore, typical side effects of IFN- $\alpha$ treatment are not expected for treatment with PEG-IFN-lambda. ${ }^{32}$ PEG-IFN-lambda has already be studied in patients with chronic hepatitis $\mathrm{B}$ and $\mathrm{C}$ mono-infection. Treatment with PEG-IFN-lambda was associated with improved or similar rates of virologic response with fewer extrahepatic adverse events than IFN- $\alpha$. However, on-treatment ALT and bilirubin flares have been observed. ${ }^{33,34}$

In a phase II study evaluating the safety, tolerability, and efficacy of PEG-IFN-lambda in CHD, 33 patients were randomized to receive either $120 \mu \mathrm{g}$ or $180 \mu \mathrm{g}$ subcutaneously for 48 weeks followed by a 24-week observation period. At the end of treatment, the primary end point of $>2 \log$ HDV RNA decline or negative HDV RNA occurred more frequently in patients treated with PEG-IFN-lambda $180 \mu \mathrm{g}$ than $120 \mu \mathrm{g}$ (7/14 vs. 4/19). Five of the 14 patients (36\%) treated with PEG-IFNlambda $180 \mu \mathrm{g}$ remained HDV RNA negative after 24 weeks of follow-up. There were fewer adverse events than during treatment with PEG-IFN- $\alpha$, but still some patients experienced hyperbilirubinemia, elevated liver enzymes and flu-like symptoms. ${ }^{35}$ Another trial investigating PEG-IFN-lambda in combination with lonafarnib over 24 weeks showed HDV RNA negativity or HDV RNA below the lower limit of quantification in 11 of 22 patients $(50 \%)$ at the end of treatment. During follow-up relapse occurred in six patients leading to $23 \%$ of patients with maintained virological response 24 weeks after the end of treatment. $^{36}$

\section{Nucleic Acid Polymers}

Nucleic acid polymers (NAPs) inhibit the assembly and release of HBsAg coated viral particles and are therefore considered as an additional antiviral approach for the treatment of HDV infection. ${ }^{37}$ REP 2139 is the first NAP, which has been studied in clinical trials focusing on HDV treatment.
In the first clinical study including 12 patients, $500 \mathrm{mg}$ of REP 2139 was given intravenously once weekly over a period of 15 weeks followed by 15 weeks of $250 \mathrm{mg}$ REP 2139 in combination with PEG-IFN- $\alpha$ followed by PEG-IFN- $\alpha$ monotherapy for 33 weeks. ${ }^{38}$ Eight patients showed HBsAg decline during REP 2139 monotherapy and 6 patients were HBsAg negative at the end of treatment. HDV RNA was significantly reduced during therapy and HDV RNA was negative in $75 \%$ of the patients $(9 / 12)$ at the end of treatment. After a follow-up of 1 year, seven and five patients were HDV RNA and HBsAg negative, respectively. Five patients developed anti-HBs by the end of the 1-year follow up. Adverse events were reported by all patients, most of them experiencing neutropenia and thrombocytopenia as well as fever, chills and peripheral hyperemia. Furthermore, asymptomatic and transient elevation of liver enzymes have been reported. Studies investigating different dosing regimens are planned.

\section{RNA Interference}

RNA interference compounds (RNAi) are short RNA molecules targeting the transcripts of viral RNA. JNJ-3989 silences all transcripts deriving from HBV cccDNA and integrated viral DNA. ${ }^{39}$ Thus, siRNAs should lead to HBsAg decline. This could also impact HDV infection. So far, only HBV monoinfected patients have been included in clinical trials. In a phase IIa study, 40 patients were enrolled to monthly receive JNJ-3989 in different doses (subcutaneously) in combination with an oral nucleotide analogue for three months. HBsAg levels showed a rapid decline during treatment and 39/40 patients had a $\geq 1.0 \log \mathrm{HBsAg}$ reduction compared to baseline. 22 patients $(22 / 39,56 \%)$ showed sustained HBsAg reduction 9 months after the last RNAi dose. Adverse events were mostly injection site reaction. Overall, short-term treatment with the RNA interference therapy, JNJ-3989, resulted in sustained hepatitis B surface antigen suppression in patients with chronic hepatitis $B$ receiving nucleotide analogue treatment. ${ }^{40}$

A phase II study to evaluate the safety and efficacy of JNJ-3989 in HBV infected patients who are co-infected with HDV is currently ongoing.

\section{Conclusion}

With the development of viral entry inhibitors, farnesylation inhibitors, viral particle formation blockers and interferon lambda, new promising treatment options are becoming available for patients with CHD. The entryinhibitor bulevirtide received conditional approval by the 
EMA this year and will change the current field of antiviral treatment of CHD. However, treatment duration is undetermined so far and prolonged treatment or even indefinite treatment are possible, especially in patients with advanced chronic liver disease. Since long-term treatment is currently investigated in the ongoing phase III study, results of this study are awaited eagerly. Data from phase II studies suggested a synergistic effect of the combination of bulevirtide with PEG-IFN- $\alpha$ but the optimal dose of bulevirtide for this concept has to be determined. Most importantly, maintained virological response was achievable with combination therapy which offers the potential for a finite antiviral treatment. Similar strategies are currently investigated for the other compounds either in combination with PEG-IFN- $\alpha$ or PEG-IFN-lambda.

The ultimate goal of CHD therapy is HBsAg clearance which also means functional cure of chronic HBV. So far, the highest rates of HBsAg loss were observed for nucleic acid polymers. To date, however, very few CHD patients have been treated with nucleic acid polymers and clearly more data is needed.

Importantly, all compounds have only been studied in patients with compensated liver disease. Since patients with CHD show high rates of advanced liver disease including a significant group of patients with decompensated liver cirrhosis, treatment options remain limited for these patients. Thus, further studies including this group of patients are necessary.

Finally, treatment strategies aiming to achieve HBsAg clearance need to be explored not only in chronic HBV but also in chronic HDV infection.

\section{Disclosure}

MC has received personal fees from AbbVie, Gilead Sciences, GSK, Janssen-Cilag, MSD Sharp \& Dohme, Novartis, Spring Bank Pharmaceuticals, Swedish Orphan Biovitrum AB (SOBI). The authors report no other conflicts of interest in this work.

\section{References}

1. Hughes SA, Wedemeyer H, Harrison PM. Hepatitis delta virus. Lancet. 2011;378(9785):73-85. doi:10.1016/S0140-6736(10)61931-9

2. Stockdale AJ, Kreuels B, Henrion MYR, et al. The global prevalence of hepatitis D virus infection: systematic review and meta-analysis. J Hepatol. 2020;73(3):523-532. doi:10.1016/j.jhep.2020.04.008

3. Alfaiate D, Clément S, Gomes D, Goossens N, Negro F. Chronic hepatitis D and hepatocellular carcinoma: a systematic review and meta-analysis of observational studies. $J$ Hepatol. 2020;73 (3):533-539. doi:10.1016/j.jhep.2020.02.030
4. Miao Z, Zhang S, Ou X, et al. Estimating the global prevalence, disease progression, and clinical outcome of hepatitis delta virus infection. J Infect Dis. 2020;221(10):1677-1687. doi:10.1093/infdis/ jiz633

5. Fattovich G, Giustina G, Christensen E, et al. Influence of hepatitis delta virus infection on morbidity and mortality in compensated cirrhosis type B. The European Concerted Action on Viral Hepatitis (Eurohep). Gut. 2000;46(3):420-426. doi:10.1136/gut.46.3.420

6. Fattovich G, Boscaro S, Noventa F, et al. Influence of hepatitis delta virus infection on progression to cirrhosis in chronic hepatitis type B. J Infect Dis. 1987;155(5):931-935. doi:10.1093/infdis/155.5.931

7. Wedemeyer H, Yurdaydin C, Dalekos GN, et al. Peginterferon plus adefovir versus either drug alone for hepatitis delta. $N$ Engl J Med. 2011;364(4):322-331. doi:10.1056/NEJMoa0912696

8. Wedemeyer H, Yurdaydin C, Hardtke S, et al. Peginterferon alfa-2a plus tenofovir disoproxil fumarate for hepatitis D (HIDIT-II): a randomised, placebo controlled, Phase 2 trial. Lancet Infect Dis. 2019;19(3):275-286. doi:10.1016/S1473-3099(18)30663-7

9. Heidrich B, Yurdaydın C, Kabaçam G, et al. Late HDV RNA relapse after peginterferon alpha-based therapy of chronic hepatitis delta. Hepatology. 2014;60(1):87-97. doi:10.1002/hep.27102

10. Wranke A, Serrano BC, Heidrich B, et al. Antiviral treatment and liver-related complications in hepatitis delta. Hepatology. 2017;65 (2):414-425. doi:10.1002/hep.28876

11. Yurdaydin C, Keskin O, Kalkan Ç, et al. Interferon treatment duration in patients with chronic delta hepatitis and its effect on the natural course of the disease. $J$ Infect Dis. 2018;217(8):1184-1192. doi:10.1093/infdis/jix656

12. Kamal H, Westman G, Falconer K, et al. Long-term study of hepatitis delta virus infection at secondary care centers: the impact of viremia on liver-related outcomes. Hepatology. 2020;72(4):1177-1190. doi:10.1002/hep.31214

13. Lampertico P, Agarwal K, Berg T, et al. EASL 2017 clinical practice guidelines on the management of hepatitis B virus infection. J Hepatol. 2017;67(2):370-398. doi:10.1016/j.jhep.2017.03.021

14. van der Meer AJ, Wedemeyer H, Feld JJ, et al. Life expectancy in patients with chronic HCV infection and cirrhosis compared with a general population. JAMA. 2014;312(18):1927-1928. doi:10.1001/ jama.2014.12627

15. Yurdaydin C, Abbas Z, Buti M, et al. Treating chronic hepatitis delta: the need for surrogate markers of treatment efficacy. J Hepatol. 2019;70(5):1008-1015. doi:10.1016/j.jhep.2018.12.022

16. Cornberg M, Lok AS-F, Terrault NA, Zoulim F. Guidance for design and endpoints of clinical trials in chronic hepatitis B - report from the 2019 EASL-AASLD HBV treatment endpoints conference $(\$)$. J Hepatol. 2020;72(3):539-557. doi:10.1016/j.jhep.2019.11.003

17. Heidrich B, Serrano BC, Idilman R, et al. HBeAg-positive hepatitis delta: virological patterns and clinical long-term outcome. Liver Int. 2012;32(9):1415-1425. doi:10.1111/j.1478-3231.2012.02831.x

18. Keskin O, Wedemeyer H, Tüzün A, et al. Association between level of hepatitis D virus RNA at week 24 of pegylated interferon therapy and outcome. Clin Gastroenterol Hepatol. 2015;13(13):2342. doi:10.1016/j.cgh.2015.05.029

19. Ni Y, Lempp FA, Mehrle S, et al. Hepatitis B and D viruses exploit sodium taurocholate co-transporting polypeptide for species-specific entry into hepatocytes. Gastroenterology. 2014;146(4):1070-1083. doi:10.1053/j.gastro.2013.12.024

20. Bogomolov P, Alexandrov A, Voronkova N, et al. Treatment of chronic hepatitis D with the entry inhibitor myrcludex B: first results of a phase Ib/IIa study. J Hepatol. 2016;65(3):490-498. doi:10.1016/ j.jhep.2016.04.016

21. Wedemeyer H, Bogomolov P, Blank A, et al. Final results of a multicenter, open-label phase $2 \mathrm{~b}$ clinical trial to assess safety and efficacy of myrcludex B in combination with tenofovir in patients with chronic HBV/HDV co-infection. J Hepatol. 2018;68:S3. doi:10.1016/S0168-8278(18)30224-1 
22. Wedemeyer H, Schöneweis K, Bogomolov PO, et al. GS-13-final results of a multicenter, open-label phase 2 clinical trial (MYR203) to assess safety and efficacy of myrcludex B in cwith PEG-interferon Alpha 2a in patients with chronic HBV/HDV co-infection. $J$ Hepatol. 2019;70(1):e81. doi:10.1016/s0618-8278(19)30141-0

23. Wedemeyer H, Schöneweis K, Bogomolov PO, et al. 48 weeks of high dose $(10 \mathrm{mg})$ bulevirtide as monotherapy or with peginterferon alfa-2a in patients with chronic HBV/HDV co-infection. J Hepatol. 2020;73: S52-S53. doi:10.1016/S0168-8278(20)30651-6

24. European Medicines Agency. Available from: https:/www.ema.europa. eu/en/medicines/human/EPAR/hepcludex. Accessed February 23, 2021.

25. Loglio A, Ferenci P, Holzmann H, et al. Myrcludex monotherapy in compensated cirrhotics with delta hepatitis: safety and effectiveness beyond two years of treatment in a real-life setting. Hepatology. 2020;72:131A-1159A.

26. Glenn JS, Watson JA, Havel CM, White JM. Identification of a prenylation site in delta virus large antigen. Science. 1992;256 (5061):1331-1333. doi:10.1126/science.1598578

27. Koh C, Canini L, Dahari H, et al. Oral prenylation inhibition with lonafarnib in chronic hepatitis D infection: a proof-of-concept randomised, double-blind, placebo-controlled phase 2A trial. Lancet Infect Dis. 2015;15(10):1167-1174. doi:10.1016/S1473-3099(15)00074-2

28. Yurdaydin C, Keskin O, Kalkan Ç, et al. Optimizing lonafarnib treatment for the management of chronic delta hepatitis: the LOWR HDV-1 study. Hepatology. 2018;67(4):1224-1236. doi:10.1002/hep.29658

29. Yurdaydin C, Kalkan C, Karakaya F, et al. Subanalysis of the LOWR HDV-2 study reveals high response rates to lonafarnib in patients with low viral loads. J Hepatol. 2018;68:S89. doi:10.1016/S01688278(18)30397-0

30. Wedemeyer H, Port K, Deterding K, et al. A phase 2 dose-escalation study of lonafarnib plus ritonavir in patients with chronic hepatitis D: final results from the lonafarnib with ritonavir in HDV-4 (LOWR HDV-4) study. J Hepatol. 2017;66(1):S24. doi:10.1016/S01688278(17)30310-0

31. Koh C, Surana P, Han T, et al. A phase 2 study exploring once daily dosing of ritonavir boosted lonafarnib for the treatment of chronic delta hepatitis - end of study results from the LOWR HDV-3 study. J Hepatol. 2017;66(1):S101-S102. doi:10.1016/S0168-8278(17)30464-6
32. Lazear HM, Schoggins JW, Diamond MS. Shared and distinct functions of type I and type III interferons. Immunity. 2019;50 (4):907-923. doi:10.1016/j.immuni.2019.03.025

33. Chan HLY, Ahn SH, Chang -T-T, et al. Peginterferon lambda for the treatment of $\mathrm{HBeAg}$-positive chronic hepatitis $\mathrm{B}$ : a randomized phase 2b study (LIRA-B). J Hepatol. 2016;64(5):1011-1019. doi:10.1016/j. jhep.2015.12.018

34. Muir AJ, Arora S, Everson G, et al. A randomized phase $2 b$ study of peginterferon lambda-1a for the treatment of chronic HCV infection. J Hepatol. 2014;61(6):1238-1246. doi:10.1016/j.jhep.2014.07.022

35. Etzion O, Hamid SS, Lurie Y, et al. PS-052-end of study results from LIMT HDV study: 36\% durable virologic response at 24 weeks post-treatment with pegylated interferon lambda monotherapy in patients with chronic hepatitis delta virus infection. J Hepatol. 2019;70(1):e32. doi:10.1016/S0618-8278(19)30058-1

36. Koh C, Da BL, Surana P, et al. A phase 2 study of lonafarnib, ritonavir and peginterferon lambda for 24 weeks: interim end-oftreatment results from the LIFT HDV study. AASLD. 2020. Late breaking abstract L08

37. Vaillant A. Nucleic acid polymers: broad spectrum antiviral activity, antiviral mechanisms and optimization for the treatment of hepatitis B and hepatitis D infection. Antiviral Res. 2016;133:32-40. doi:10.1016/j.antiviral.2016.07.004

38. Bazinet M, Pântea V, Cebotarescu V, et al. Safety and efficacy of REP 2139 and pegylated interferon alfa-2a for treatment-naive patients with chronic hepatitis $B$ virus and hepatitis $D$ virus co-infection (REP 301 and REP 301-LTF): a non-randomised, open-label, phase 2 trial. Lancet Gastroenterol Hepatol. 2017;2 (12):877-889. doi:10.1016/S2468-1253(17)30288-1

39. Smolders EJ, Burger DM, Feld JJ, Kiser JJ. Review article: clinical pharmacology of current and investigational hepatitis B virus therapies. Aliment Pharmacol Ther. 2020;51(2):231-243. doi:10.1111/apt.15581

40. Gane E, Locarnini S, Lim TH, et al. Short-term treatment with RNA interference therapy, JNJ-3989, results in sustained hepatitis B surface antigen suppression in patients with chronic hepatitis B receiving nucleos(t)ide analogue treatment. $J$ Hepatol. 2020;73: S20. doi:10.1016/S0168-8278(20)30597-3

\section{Publish your work in this journal}

The Journal of Experimental Pharmacology is an international, peerreviewed, open access journal publishing original research, reports, reviews and commentaries on all areas of laboratory and experimental pharmacology. The manuscript management system is completely online and includes a very quick and fair peer-review system. Visit http://www.dovepress.com/testimonials.php to read real quotes from published authors. 